sinus between the cornea and iris, and was not imbedded in the latter. The iris in its neighbourhood seemed to be slightly muddy, but this appearance might have been due to a condicion of the cornea or aqueous humour. The ocular conjunctiva around this part of the cornea was very hyperemic, and it had slightly encroached upon the cornea. There was also localised ciliary congestion. The attacks of irritation could now be accounted for on the supposition that the active exercise-tennis, shooting, riding, etc.-in which Mr. T. engaged caused some slight movement of the glass; and he could now recollect that each fresh attack had followed exercise.

A quiet life was advised, and in two months the muddiness of the iris and the ciliary congestion had disappeared, and much of the conjunctival redness had gone.

On September 12th, 1887, the operation was performed; and I may here thank Dr. Fox, of Philadelphia, an old Moorfields friend, for the good advice and able assistance he gave me. Eserine having been freely applied, in order to cause firm contraction of the iris, and the eye placed thoroughly under the influence of a 20 per cent. solution of cocaine, a horizontal, purely corneal incision was made by puncture and counter-puncture with a Graefe's knife across the front of the cornea, at the junction of the lower third with the upper two-thirds, the aqueous being allowed to escape slowly. A curette was then introduced between the lips of the wound and passed on in front of the iris to a position behind the foreign body, in order to fix it. This being done, a pair of fine forceps was introduced to the bottom of the anterior chamber, and the glass, being seized at the first attempt, was removed entire. At the moment of withdrawal a speck of blood appeared on the surface of the iris, evidently being due to its having been pricked by a fine needle-like point which projected from one of the ends of the glass. Iced compresses were applied, and the eye made an uninterrupted good recovery, though, in spite of frequent instillations of eserine, a slight adhesion took place between the surface, not the pupillary margin, of the iris and the outer part of the corneal incision.

Two months after the removal all conjunctival redness had gone; the pupil was almost regular, and had increased in size to $3 \frac{1}{2} \mathrm{~mm}$. Vision $\mathbf{a}_{0}^{\mathrm{a}} 4$ letters, J. i.

The glass measures in its greatest length $43 \mathrm{~mm}$., in its shortest length $33 \mathrm{~mm}$. It is $1 \mathrm{~mm}$. in breadth, and $\frac{3}{4 m}$. in thickness.

\section{A NOTE ON THE CONDITION OF THE BLOOD IN MALARIA.}

BY J. F. FVANS, SURGEON, I.M.S., MANDALAY.

Malarial fevers have naturally always obtained a great deal of attention from medical men throughout the Indian Empire. In 1886, according to Surgeon-General Thomson's report, one-fourth of the admissions to the European military hospitals of the Bombay Presidency were for this disease. But, while the mortality is rarely high, the deloility that results from repeated attacks is a much more serious matter. Englishmen who have been living in a malurious climate occasionally suffer from ague after their arrival home, pointing to some effect on the tissues persisting subsequent to exposure. An officer who had been some years in India told me that his first attack of ague was when taking his first leave home.

It may le that malarial poisoning is a gradual process, and that a condition of semi-tolerance is established, and that the chill or exposure, of which Dr. Oldham writes, is the immediate exciting cause. In 1879, Klebs and Tommasi-Crudeli professed to have successfully inoculated rubbits with malarial fever. and to have discovered the bacillus malarie : their experiments do not seem to have been contirmel by other olservers. Marchiafava and Celli, in 1885 , published a detailed account of the changes observed by them in malarial blood, with which the later researches of Dr. Litveran in great mensure coincide vide British Medrcal Jotrisal. October 29th, 1887). There has of late, in the military hospitals of Mandalay, been a wide field for research into the pathological changes induced by malaria. I have never examined a single specimen of blood taken from a patient suffering from ague or remittent fever without finding some definite change.

The following are the changes observed by me:

1. In health the red blood cells are with difficulty stained by an aniline dye, but, after even a single attack of ague, the red blood cells can be readily stained by an aniline solution of gentian violet; a small area, however, in most being left unstained. This unstained area is not constant in size, nor in its position in the corpuscle, in some involving nearly the whole corpuscle, in others 80 small as to be easily ignored. In specimens of unstained blood I have recognised the hyaline material replacing the hæmoglobin of the red corpuscles, as described by Marchiafava and Celli, who state that this hyaline material can be readily stained with methyl blue; but hitherto I have not been successful in obtaining this reaction. The globule of new hyaline matter in the red corpuscles can be seen slowly to change its shape from time to time, which Marchiufava and Celli pointed out. Whether this hyaline material coincides with the unstuined area, I am not at present able to d $\theta$ cide. In cases of malarial cachexia, the softening and crenation of the red corpuscles is a marked feature; in such cases, it is difficult to find in a specimen of blood a corpuscle with normal outline. This crenation is certainly more marked in the blood of natives than in that of Europeans, and may be due in part to the deficiency of nitrogenous matter in their diet. It is due probably to a gradual loss of the cell contents, and may serve to account for the intense anæmia that occurs in malarial cachexia. The red corpuscles are not only altered in structure by this disease, but are absolutely destroyed in the process; they can be seen in every stage of destruction, from mere erosion of the margin to almost complete obliteration, a thin semilunar rim of pellicle being left. As might be expected in blood undergoing a process of slow deterioration, small free masses of pigment are often met with.

2. In addition to the structural changes already mentioned in tlie blood-corpuscles, I have found free spherical bodies in the serum and in the corpuscles. These spherical bodies are possessed of movement, both rotatory and from place to place. They are of two perfectly distinct kinds, and I shall, for purposes of description, describe ther. as nucleated and non-nucleated, although not considering the nuclear body of the nature of a nucleus :

$a$. The non-nucleated spherical bodies are dark in colour, of the same consistence throughout, and about ${ }^{2}$ th th th the diameter of a red corpuscle (that is to say, $0.2 \mu$ to $0.3 \mu$ in diameter). They are to be met with either moving freely about in the serum, or else stationary inside the red corpuscles; when immured in a red corpuscle they as a rule remain fixed, but rotatory movements are to be observed sometimes. Three or four of these bodies generally occur in the same corpuscle together, beneath the pellicle, at one point; they frequently occur in the corpuscles undergoing the hyaline degeneration noted by Marchiafava and Celli, and also in those cells where this degeneration cannot be distinguished. I have never seen any of these organisms inside a white blood-corpuscle. I have found them in the serum and red cells of the blood of men suffering from beri-beri; and similarly in the serum and red cells of the blood of horses suffering from surrah, in company with Surgeon IIendley, who tells me that he has observed similar bodies in the blood of rats, and of a dog ailing with fever. More numerous and more freely movable than the nucleated spherical bodies, it is only possible to suggest that they may have some relation to the nuclear-like bodies of the latter, although up to the present this has not been traced.

$b$. The nucleated spherical bodies are of a greenish-yellow tint, circular single contour. brighter than the red corpuscles, and with a highly refructive out line; varying slightly in size, they are about th th to th the diameter of a red blood corpuscle (about $I_{\mu}$ to $0.7 \mu$ ). The nuclear-like body can be easily distinguished inside the sphere, which is often little more than an enclosing capsule. Like the spherical body in which it is enclosed, this nucleus has free rotatory movement.

The mucleated, like the non-nucleated bodies, have movements rotatory and from place to place, though not so freely as the latter; ther are also not so numerous, but, like them, are found floating freely in the plasma and stationary in the corpuscles. They usually occur singly in the blood cells, and I have seen them occasionally in the white hlond cells as well as in the red. They often occur with the non-nucleated in the same red corpuscle; but I have never found them in corpuscles undergoing the hyaline degeneration My first arquintance with these nucleated spherical bodies was in the blood of horses suffering from surrah, where they occur like the non-nucleated. I have not seen them, however, in the blood of beri-beri, where the non-nucleated occur, and in which disease, on two occasions quite recently, I have found a streptococcus, in chains of three cocci each, not very unlike the cocci occurring in kumri. The appearance of these nucleated spherical bodies would justify the assumption of their being monococci. I have not jet, however, succeeded in staining them. Accordingly, 
without this and cultivation on nutrient media, and the reproduction of the disease from culture, it would be unwise to pronounce as to their nature.

The larger spherical bodies, described by MI. Laveran as slightly larger than red blood cells, I have not hitherto seen; but $1 \mathrm{am}$ inclined to believe that, what are described by him as the larger spheroids are red blood cells invaded by the parasitical elements I have observed. The white blood-corpuscles in malaria are not increased; they are apparently little affected by the disease, being invaded occasionally by the nucleated spheroids; and, when studded with pigment granules, as sometimes occurs, they present the appearance indicated by $\mathbf{M}$. Laveran as the "leucocytes mélaniferes."

\section{THE CIIEMICAL INCOMPATIBILITY OF ANTISEPTIC AGENTS.}

BY ROBERT BOXALL, M.D., M.R.C.l'.,

Physician to the General Lying-in and to the Samaritan Free Hospitals.

THE necessity of employing antiseptic agents in solution of definite strength will be, $I$ presume, on all hands conceded; for, if the solution be too attenuated, the object in view will fail in its accomplishment, and, if too concentrated, considerable damage will in many cases be wrought, not only locally on the tissues to which the application is made, but also on the body generally as the result of absorption. The borderland between safety and success is, in many instances, a very narrow one. The possibility of reducing the strength of the solution, or of altering its nature through the chemical incompatibility of the materials employed, has hitherto received but little attention. The important practical bearing which this may exert on their efficiency as antiseptics must prove my apology for drawing attention to the matter.

By way of example, I have selected five of the more important antiseptic agents in general use, and, for ready reference as to the incompatibilities of each, the results of the experiments are presented in a tabular form, showing the action not only of these agents on one another, but also of certain lubricants with which they are frequently combined and brought into contact, and of soap with which they are apt to be contaminated in the process of washing and disinfecting the hands and instruments.

In view of the practical utility of these observations, the experiments were made, not with concentrated materials, but with solutions of the strengths usually employed in practice, and were carried out at temperatures not exceeding that of the body.
1. Corrosive Sublimate Solution (Perchloride of Mercury)

. Carbolic Solution (Phenol) $\ldots \ldots$ of Potassium)

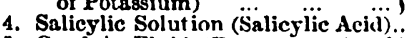

5. Condy's Fluid (Permanganate of $j$ Potassium

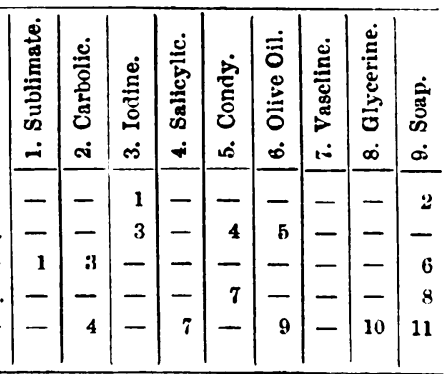

The following incompatibilities were observed :

1. Corrosive Sublimate and Iodine.-No precipitate of mercuric iodide is at any stage of the admixture formed. A small addition of sublimate solution fixes the free iodine. as may be seen by the immediate bleaching of the iodine solution, and confirmed by the subsequent addition of starch paste, which produces no blue coloration. One part by volume of sublimate solution $(1$ in 1,000$)$ is just sufficient to fix the whole of the free iodine in 4 parts by volume of iodine solution (tr. iod. B.P. $3 \mathrm{j}$ in $\mathrm{Oj}$ ). N.B.-This forms a rough and ready test for the strength of sublimute solutions.

2. Corrosive Sublimate and Suap.-An insoluble soap is produced even when a neutral soap solution is used. This is of special importance in consideration of the small admixture with soap which is required to throw down the whole of the mercury from solutions of the strength usually employed.

3. Carbolic and Iodine.-An exceedingly small admixture with phenol is sufficient to fix the whole of the free iodine as in (1). One part by volume of carbolic solution (1 in 20) removes the whole of the free iodine from 2,000 parts by volume of iodine solution of the strength indicated above.

4. Carbolic and Condy.-This is perhaps the most generally recognised of these incompatibilities. Admixture with phenol immediately turns permanganate brown.

5. Carbolic and Olive Oil.-This is of importance and of special interest when taken in conjunction with the researches of Koch, of Berlin, who has shown that bacillus spores are capable of living and developing after having been immersed in carbolised oil (1 in 20) for 4 months. The oil appears to enter into some combination with and to fix the phenol. If a drop of tr. ferri perchlor. B.P. be shaken up in a test tube with carbolised oil ( 1 in 20$)$ no change is found to have been produced in the iron as it gravitates to the bottom. Moreover, if carbolised oil be shaken up with a few drops of water, the water allowed to separate out at the bottom of the tube and a drop of iron solution conveyed into it, the characteristic purple coloration with phenol is not produced unless the shaking has been very prolonged and energetic, and then only to a slighit degree. By strongly heating the carbolised oil phenol is again set free, and the above reaction can then be obtained.

6. Iodine and Soap.-No action is produced by a neutral soap solution, but ordinary soap, which contains an excess of alkali, at once removes the free iodine.

7. Salicylic Acid and Condy.-A very dilute salicylic acid solution ( 1 in 800) slowly removes the colour from permanganate.

8. Salicylic Acid and Soap.-A drop of dilute salicylic acid solution gives a white precipitate even when a neutral soap solution is emplojed.

9. Condy and Olive Oil.- When permanganate solution is shaken up with olive oil its violet colour is changed to brown.

10. Condy and Glycerine.- When permanganate solution is added to glycerine its colour slowly changes.

11. Condy and Soap.-This incompatibility is also generally recognised. Soap, even when a neutral solution is employed, readily turns permanganate brown.

I do not pretend to any precise knowledge of the bodies produced, some of which may, for all I know, possess powerful antiseptic properties. But until this point is settled by direct observation, when chemical incompatibility exists, the antiseptic properties of the original solution must be regarded as weakened, if not wholly destroyed.

The moral conreyed by the above experiments is obvious : avoid as far as possible the admixture of antiseptic agents and their contamination with lubricants and with soap when incompatibility exists. For instance, in employing corrosive sublimate it is advisable to use the same solution for disinfecting the hands (carefully aroiding contamination with soap) and for cleansing instruments ${ }^{1}$ as for irrigating the parts, to employ a mercurialised lubricant and to use alembroth dressings. If for any reason it becomes requisite to substitute one antiseptic agent for another, a second should be chosen which is not incompatible with the first, and the same precautions should be observed throughout the series.

The above observations deal with one phase only of the subject. I am content to leave to more able hands the elaboration of further details. The second question-the chemical nature of the bodies produced -is for the chemist to answer; the third point-the antiseptic value of these bodies-still remains for the germiculturist to determine. When these points have been settled and acted upon, less scepticism as to the value of antiseptic agents mny be looked for. The fuult lies not so much with the antiseptics themselves as with the unscientific method in which they are often employed.

1 Here, again, a caution is requisite, for copper and steel, unless nickel plated. are apt to decompose the solution and to cause precipitation of the mercury in a free state.

TestruoniaL.-Dr. Williams, who was recently compelled to resign his appointment of medical superintendent of the Sussex Lunatic Asylum at Hayward's Heath in consequence of ill-health, has been presented by upwards of one hundred members of the staff of that institution with an illuminated address in the form of an album as an expression of their apprecintion and regard.

At a meeting of the Committee of the Home and Infirmary for Sick Children, Lower Sydenham, S.E., on April 2nd, a unanimous vote of thanks was passed to Dr. Spencer Smyth, F.R.C.S. for valuable services rendered to the institution for so long a time as Honorary Surgeon and Consulting Surgeon. Dr. Smyth has gono to reside at Bournemouth. 